\title{
A Study on Strongly Almost Convergent and Strongly Almost Null Binomial Double Sequence Spaces
}

\author{
Sezer Erdem ${ }^{1 *}$ and Serkan Demiriz ${ }^{2}$ \\ ${ }^{1}$ Battalgazi Farabi Anatolian Imam Hatip High School, Malatya, Turkey \\ ${ }^{2}$ Department of Mathematics, Tokat Gaziosmanpaşa University, Tokat, Turkey \\ * Corresponding author
}

\section{Article Info}

Keywords: 4d binomial matrix, Double sequence space, Duals, Matrix transformations, $R H$ regularity

2010 AMS: 40C05, 46 A45

Received: 27 August 2021

Accepted: 12 November 2021

Available online: 1 December 2021

\begin{abstract}
The 4 dimensional (4d) binomial matrix and its domains on the classical double sequence spaces $\mathscr{L}_{p}, \mathscr{M}_{u}, \mathscr{C}_{P}, \mathscr{C}_{b P}, \mathscr{C}_{r}, \mathscr{C}_{f}$ and $\mathscr{C}_{f_{0}}$ have been described and examined by Demiriz and Erdem in the papers [1]-[3]. In this article, we describe two double sequence spaces with the aid of the aforementioned matrix and study some properties of these. After giving inclusion relations, we compute $\alpha-\beta(b p)-$ and $\gamma$-duals and give some new matrix classes related them.
\end{abstract}

\section{Introduction}

The function $F$ defined by $F: \mathbb{N} \times \mathbb{N} \rightarrow \zeta,(i, j) \mapsto F(i, j)=u_{i j}$ is called as double sequence where $\zeta$ denotes any nonempty set and $\mathbb{N}=\{0,1,2, \ldots\} . \Omega$ represents the vector space of all complex valued double sequences. $\mathscr{M}_{u}, \mathscr{C}_{P}, \mathscr{C}_{r}$ and $\mathscr{L}_{p}(0<p<\infty)$ are the spaces of all bounded, convergent in the Pringsheim's sense (or shortly $P$-convergent), regularly convergent and $p$-absolutely summable double sequences, respectively. If any $u=\left(u_{i j}\right) \in \Omega$ is $P$-convergent to a limit point $L$, it is stated by $P-\lim _{i, j \rightarrow \infty} u_{i j}=L$. It is worth mentioning that $P$-convergence does not require boundedness in double sequences. The bounded sequences which are also $P$-convergent are indicated by $\mathscr{C}_{b P}$. It is also significant to remember that the space $\mathscr{L}_{u}$ which was described by Zeltser [4] is the special case of the space $\mathscr{L}_{p}$ for $p=1$.

Throughout this article, it is used the summation $\sum_{i, j}$ instead of $\sum_{i=0}^{\infty} \sum_{j=0}^{\infty}$ and $\vartheta \in\{p, b P, r\}$. With the notation of Zeltser [4], we describe the double sequences $e^{k l}=\left(e_{i j}^{k l}\right)$ and $e$ by $e_{i j}^{k l}=1$ if $(k, l)=(i, j)$ and $e_{i j}^{k l}=0$ for other cases, and $e=\sum_{k, l} e^{k l}$ for every $i, j, k, l \in \mathbb{N}$. If $d_{k l i j}=0$ for $i>k$ or $j>l$ or both for every $k, l, i, j \in \mathbb{N}$, it is said that $D=\left(d_{k l i j}\right)$ is a triangular matrix and also if $d_{k l k l} \neq 0$ for every $k, l \in \mathbb{N}$, then the $4 \mathrm{~d}$ matrix $D$ is called triangle.

Now, we shall deal with the matrix mapping. Let us consider double sequence spaces $\Psi$ and $\Lambda$ and the $4 \mathrm{~d}$ complex infinite matrix $D=\left(d_{k l i j}\right)$. If for every $u=\left(u_{i j}\right) \in \Psi,(D u)_{k l}=\vartheta-\sum_{i, j} d_{k l i j} u_{i j}$ is exists and is in $\Lambda$, then it is said that $D$ is a matrix mapping from $\Psi$ into $\Lambda$ and is written as $D: \Psi \rightarrow \Lambda$.

Let $(\Psi, \Lambda)=\left\{D=\left(d_{k l i j}\right) \mid D: \Psi \rightarrow \Lambda\right\}$. Here, $D \in(\Psi, \Lambda)$ if and only if $D_{k l} \in \Psi^{\beta(\vartheta)}$ and $D u \in \Lambda$ for all $u \in \Psi$, where $D_{k l}=\left(d_{k l i j}\right)_{i, j \in \mathbb{N}}$ for every $k, l \in \mathbb{N}$.

The domain $\Psi_{D}^{(\vartheta)}$ of $D$ in a double sequence space $\Psi$ consists of whose $D$-transforms are in $\Psi$ is defined by the following way:

$$
\Psi_{D}^{(\vartheta)}:=\left\{u=\left(u_{i j}\right) \in \Omega: D u:=\left(\vartheta-\sum_{i j} d_{k l i j} u_{i j}\right)_{k, l \in \mathbb{N}} \text { exists and is in } \Psi\right\} \text {. }
$$


In the past, many authors were interested in double sequence spaces. Now, let us give some information about these studies. In her doctoral dissertation, Zeltser [5] has fundamentally examined the topological structure of double sequences. Recently, Altay and Başar [6] have been described the spaces $\mathscr{B} \mathscr{S}, \mathscr{B} \mathscr{S}(t), \mathscr{C} \mathscr{S}_{P}, \mathscr{C} \mathscr{S}_{b P}, \mathscr{C} \mathscr{S} r$ and $\mathscr{B} \mathscr{V}$ of double series. After that, Talebi [7] defined and examined the space $\mathscr{E}_{p}^{r, s}$ for $1 \leq p<\infty$ and also Yeșilkayagil and Bașar [8] examined for $0<p<1$ where $\mathscr{E}_{p}^{r, s}=\left\{u=\left(u_{i j}\right): E(r, s) u \in \mathscr{L}_{p}\right\}$. Here, $E(r, s)$ indicates the Euler mean. More recently, Tug [9]-[11] have defined and examined some domains of the $4 \mathrm{~d}$ matrix $B(r, s, t, u)$.

On the other hand, Bişgin [12,13] have introduced the sequence spaces $b_{0}^{r, s}, b_{c}^{r, s}, b_{p}^{r, s}$ and $b_{\infty}^{r, s}$ of single sequences whose $2 \mathrm{~d}$ binomial matrix $B^{r, s}$-transforms are convergent to zero, convergent, absolutely $p$-summable and bounded, respectively. After that in [14], Bişgin have been examined the domains of $B^{r, s}$ on $f$ and $f_{0}$. Here, $f$ and $f_{0}$ symbolize the spaces of every almost convergent and almost null single sequences, respectively.

A generalization for convergence of a double sequence is almost convergence was firstly introduced by Mòricz and Rhoades [15]. It is said that $u \in \Omega$ is almost convergent if

$$
P-\lim _{\rho, \rho^{\prime}} \sup _{k, l>0}\left|\frac{1}{(\rho+1)\left(\rho^{\prime}+1\right)} \sum_{i=k}^{k+\rho} \sum_{j=l}^{l+\rho^{\prime}} u_{i j}-L\right|=0
$$

and stated by $f_{2}-\lim u=L$. Every almost convergent $u \in \Omega$ are included by $\mathscr{C}_{f}$ which is defined by the following way:

$$
\mathscr{C}_{f}=\left\{u=\left(u_{i j}\right) \in \Omega: \exists L \in \mathbb{C} \ni \quad P-\lim _{\rho, \rho^{\prime}} \sup _{k, l>0}\left|\frac{1}{(\rho+1)\left(\rho^{\prime}+1\right)} \sum_{i=k}^{k+\rho} \sum_{j=l}^{l+\rho^{\prime}} u_{i j}-L\right|=0, \text { uniformly in } k, l\right\} .
$$

Moreover, the space of almost null double sequences $\mathscr{C}_{f_{0}}$ is obtained from $\mathscr{C}_{f}$ by taking $L=0$.

It is significant to say that the convergence of a double sequence does not require its almost convergence. However, the inclusion $\mathscr{C}_{b P} \subset \mathscr{C}_{f} \subset \mathscr{M}_{u}$ is valid.

With the notion Başarır [16], it is said that $u=\left(u_{k l}\right) \in \Omega$ is strongly almost convergent to a limit point $L_{1}$ if

$$
P-\lim _{\rho, \rho^{\prime}} \sup _{k, l>0} \frac{1}{(\rho+1)\left(\rho^{\prime}+1\right)} \sum_{i=k}^{k+\rho} \sum_{j=l}^{l+\rho^{\prime}}\left|u_{i j}-L_{1}\right|=0, \quad \text { uniformly in } k, l \in \mathbb{N} .
$$

In that case, this stuation is shown by $\left[f_{2}\right]-\lim u=L_{1}$.

Every strongly almost convergent $u \in \Omega$ are included by $\left[\mathscr{C}_{f}\right]$ which is defined by the following way:

$$
\left[\mathscr{C}_{f}\right]=\left\{u=\left(u_{i j}\right) \in \Omega: \exists L_{1} \in \mathbb{C} \ni \quad P-\lim _{\rho, \rho^{\prime}} \sup _{k, l>0} \frac{1}{(\rho+1)\left(\rho^{\prime}+1\right)} \sum_{i=k}^{k+\rho} \sum_{j=l}^{l+\rho^{\prime}}\left|u_{i j}-L_{1}\right|=0, \text { uniformly in } k, l\right\} .
$$

Furthermore, the space of strongly almost null double sequences $\left[\mathscr{C}_{f_{0}}\right]$ is obtained from $\left[\mathscr{C}_{f}\right]$ by taking $L_{1}=0$. Between the mentioned spaces, the inclusion relations $\mathscr{C}_{b P} \subset\left[\mathscr{C}_{f_{0}}\right] \subset\left[\mathscr{C}_{f}\right] \subset \mathscr{M}_{u}$ and $\mathscr{C}_{b P} \subset \mathscr{C}_{f_{0}} \subset \mathscr{C}_{f} \subset \mathscr{M}_{u}$ strictly hold. Moreover, the spaces $\left[\mathscr{C}_{f}\right]$ and $\left[\mathscr{C}_{f_{0}}\right]$ are Banach spaces with norm

$$
\|u\|_{\left[\mathscr{C}_{f}\right]}=\sup _{\rho, \rho^{\prime}, k, l \in \mathbb{N}} \frac{1}{(\rho+1)\left(\rho^{\prime}+1\right)} \sum_{i=k}^{k+\rho} \sum_{j=l}^{l+\rho^{\prime}}\left|u_{i j}\right| .
$$

For further information about single and double sequence spaces and related topics, the reader may refer to some of the papers [17]-[39] and references therein.

Our main purpose in this article is to investigate the domains of $4 \mathrm{~d}$ binomial matrix on the spaces $\left[\mathscr{C}_{f}\right]$ and $\left[\mathscr{C}_{f_{0}}\right]$.

\section{Strongly almost convergent binomial double sequence spaces}

Let $r, s$ and $r+s$ are nonzero real numbers. We have been defined the $4 \mathrm{~d}$ binomial matrix $B^{(r, s)}=\left(b_{k l i j}^{r, s}\right)$ of orders $r, s$ in [1] as follows:

$$
b_{k l i j}^{r, s}:=\left\{\begin{array}{cl}
\frac{1}{(r+s)^{k+l}}\left(\begin{array}{c}
k \\
i
\end{array}\right)\left(\begin{array}{c}
l \\
j
\end{array}\right) s^{k+j-i} r^{l+i-j} & , \quad 0 \leq i \leq k, 0 \leq j \leq l \\
0 & , \quad \text { otherwise }
\end{array}\right.
$$

for every $k, l, i, j \in \mathbb{N}$. As can be understood from its definition, $B^{(r, s)}$ is a triangle. In that case, we write the $B^{(r, s)}$-transform of $u \in \Omega$ as

$$
v_{k l}:=\left(B^{(r, s)} u\right)_{k l}=\sum_{i, j}^{k, l} \frac{1}{(r+s)^{k+l}}\left(\begin{array}{c}
k \\
i
\end{array}\right)\left(\begin{array}{l}
l \\
j
\end{array}\right) s^{k+j-i} r^{l+i-j} u_{i j}
$$


for every $k, l \in \mathbb{N}$. We will assume unless stated otherwise that the double sequences $u=\left(u_{i j}\right)$ and $v=\left(v_{i j}\right)$ are connected with the relation (2.2) and $r, s$ and $r+s$ are nonzero real numbers. We would like touch on a point, when it is chosen $r+s=1$, $B^{(r, s)}$ is reduced to the $4 \mathrm{~d}$ Euler matrix $E(r, s)$. So, our matrix $B^{(r, s)}$ generalizes the $E(r, s)$. Consider that the $4 \mathrm{~d}$ unit matrix $I=\left(\delta_{k l i j}\right)$ defined by

$$
\delta_{k l i j}=\left\{\begin{array}{cc}
1 \quad, & (k, l)=(i, j) \\
0, & \text { otherwise }
\end{array}\right.
$$

From the equality

$$
\delta_{k l i j}=\sum_{m, n} b_{k l m n}^{r, s} c_{m n i j}^{r, s}
$$

one can see that the inverse $\left\{B^{(r, s)}\right\}^{-1}=C^{(r, s)}=\left(c_{k l i j}^{r, s}\right)$ as

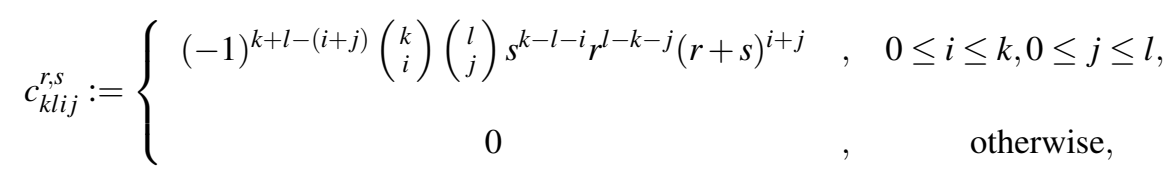

for every $k, l, i, j \in \mathbb{N}$.

A $4 \mathrm{~d}$ matrix D is said to be RH-regular if it maps every bounded $P$-convergent sequence into a $P$-convergent sequence with the same $P$-limit $[22,32]$. In [1], it was proven that $4 \mathrm{~d}$ binomial matrix described by (2.1) is RH-regular for $r . s>0$. In the rest of the study, it will be assumed that $r . s>0$.

Now, we introduce the sets $\mathscr{B}_{[f]}^{r, s}$ and $\mathscr{B}_{\left[f_{0}\right]}^{r, s}$ by

$$
\begin{aligned}
\mathscr{B}_{[f]}^{r, s} & =\left\{u=\left(u_{i j}\right) \in \Omega: \exists L \in \mathbb{C} \ni P-\lim _{\rho, \rho^{\prime}} \sup _{k, l>0} \frac{1}{(\rho+1)\left(\rho^{\prime}+1\right)} \sum_{i=k}^{k+\rho} \sum_{j=l}^{l+\rho^{\prime}}\left|\left(B^{(r, s)} u\right)_{i j}-L\right|=0, \text { uniformly in } k, l\right\}, \\
\mathscr{B}_{\left[f_{0}\right]}^{r, s} & =\left\{u=\left(u_{i j}\right) \in \Omega: P-\lim _{\rho, \rho^{\prime}} \sup _{k, l>0} \frac{1}{(\rho+1)\left(\rho^{\prime}+1\right)} \sum_{i=k}^{k+\rho} \sum_{j=l}^{l+\rho^{\prime}}\left|\left(B^{(r, s)} u\right)_{i j}\right|=0, \text { uniformly in } k, l\right\} .
\end{aligned}
$$

Theorem 2.1. The sets $\mathscr{B}_{[f]}^{r, s}$ and $\mathscr{B}_{\left[f_{0}\right]}^{r, s}$ are linear spaces.

Proof. Since it is easy to see, we omit it.

Theorem 2.2. The sequence spaces $\mathscr{B}_{[f]}^{r, s}$ and $\mathscr{B}_{\left[f_{0}\right]}^{r, s}$ are Banach spaces with the norm

$$
\|u\|_{\mathscr{B}_{[f]}^{r, s}}=\sup _{\rho, \rho^{\prime}, k, l \in \mathbb{N}} \frac{1}{(\rho+1)\left(\rho^{\prime}+1\right)} \sum_{i=k}^{k+\rho} \sum_{j=l}^{l+\rho^{\prime}}\left|\left(B^{(r, s)} u\right)_{i j}\right| .
$$

Proof. Since it can be similarly proven for the space $\mathscr{B}_{\left[f_{0}\right]}^{r, s}$, it will be proven for $\mathscr{B}_{[f]}^{r, s}$.

Consider any cauchy sequence $u^{(m)}=\left\{u_{i j}^{(m)}\right\}_{i, j \in \mathbb{N}} \in \mathscr{B}_{[f]}^{r, s}$. In that case, for $\varepsilon>0$ there exists a $N \in \mathbb{N}^{+}$such that

$$
\left\|u^{(m)}-u^{(n)}\right\|_{\mathscr{B}}^{r, s]}=\sup _{\rho, \rho^{\prime}, k, l \in \mathbb{N}} \frac{1}{(\rho+1)\left(\rho^{\prime}+1\right)} \sum_{i=k}^{k+\rho} \sum_{j=l}^{l+\rho^{\prime}}\left|\left(B^{(r, s)} u^{(m)}\right)_{i j}-\left(B^{(r, s)} u^{(n)}\right)_{i j}\right|<\varepsilon
$$

for all $m, n>N$. Thus, it is concluded from (2.4), $\left\{\left(B^{(r, s)} u^{(m)}\right)_{i j}\right\}$ is also Cauchy in $\left[\mathscr{C}_{f}\right]$. Since, $\left[\mathscr{C}_{f}\right]$ is a Banach space, we can write

$$
\left\{\left(B^{(r, s)} u^{(m)}\right)_{i j}\right\} \longrightarrow\left\{\left(B^{(r, s)} u\right)_{i j}\right\}
$$

as $m \rightarrow \infty$ and using these infinitely many limit points, we can define double sequence $\left\{\left(B^{(r, s)} u\right)_{i j}\right\}$.

Now, by taking the limit as $n \rightarrow \infty$ on (2.4), we have

$$
\sup _{\rho, \rho^{\prime}, k, l \in \mathbb{N}} \frac{1}{(\rho+1)\left(\rho^{\prime}+1\right)} \sum_{i=k}^{k+\rho} \sum_{j=l}^{l+\rho^{\prime}}\left|\left(B^{(r, s)} u^{(m)}\right)_{i j}-\left(B^{(r, s)} u\right)_{i j}\right|<\varepsilon
$$

for all $\varepsilon>0, m>N$ and $i, j \in \mathbb{N}$. 
Furthermore, since $u^{(m)} \in \mathscr{B}_{[f]}^{r, s}$, it is clear that $B^{(r, s)} u^{(m)} \in\left[\mathscr{C}_{f}\right]$ and

$$
\sup _{\rho, \rho^{\prime}, k, l \in \mathbb{N}} \frac{1}{(\rho+1)\left(\rho^{\prime}+1\right)} \sum_{i=k}^{k+\rho} \sum_{j=l}^{l+\rho^{\prime}}\left|\left(B^{(r, s)} u^{(m)}\right)_{i j}\right| \leq M
$$

for a positive real number $M$. Now, we can say by taking supremum over $\rho, \rho^{\prime}, k, l \in \mathbb{N}$ on the inequality

$$
\begin{aligned}
\frac{1}{(\rho+1)\left(\rho^{\prime}+1\right)} \sum_{i=k}^{k+\rho} \sum_{j=l}^{l+\rho^{\prime}}\left|\left(B^{(r, s)} u\right)_{i j}\right| & \leq \frac{1}{(\rho+1)\left(\rho^{\prime}+1\right)} \sum_{i=k}^{k+\rho} \sum_{j=l}^{l+\rho^{\prime}}\left|\left(B^{(r, s)} u^{(m)}\right)_{i j}-\left(B^{(r, s)} u\right)_{i j}\right| \\
& +\frac{1}{(\rho+1)\left(\rho^{\prime}+1\right)} \sum_{i=k}^{k+\rho} \sum_{j=l}^{l+\rho^{\prime}}\left|\left(B^{(r, s)} u^{(m)}\right)_{i j}\right|<\varepsilon+M
\end{aligned}
$$

that $B^{(r, s)} u \in\left[\mathscr{C}_{f}\right]$, that is $u \in \mathscr{B}_{[f]}^{r, s}$. Thus, it is concluded that $\mathscr{B}_{[f]}^{r, s}$ is a Banach space with the norm $\|u\|_{\mathscr{B}_{[f]}^{r, s}}$ defined by $(2.3)$.

Theorem 2.3. The double sequence spaces $\mathscr{B}_{[f]}^{r, s}$ and $\mathscr{B}_{\left[f_{0}\right]}^{r, s}$ are linearly norm isomorphic to the spaces $\left[\mathscr{C}_{f}\right]$ and $\left[\mathscr{C}_{f_{0}}\right]$, respectively.

Proof. Because it can be similarly shown for the space $\mathscr{B}_{[f]}^{r, s}$, we give the proof only for $\mathscr{B}_{\left[f_{0}\right]}^{r, s}$. For the claim of theorem, we must see that there is a linear bijection which preserves the norm from one to the other for the spaces $\mathscr{B}_{\left[f_{0}\right]}^{r, s}$ and $\left[\mathscr{C}_{f_{0}}\right]$.

For this purpose, let us take the map $T: \mathscr{B}_{\left[f_{0}\right]}^{r, s} \rightarrow\left[\mathscr{C}_{f_{0}}\right], u \mapsto v=T u=B^{(r, s)} u$. The linearity of $T$ is clear. Consider the equality $T u=\theta$ which yields us that $u_{i j}=0$ for every $i, j \in \mathbb{N}$. So, $u=\theta$ and therefore, $T$ is injective. Let us consider $v \in\left[\mathscr{C}_{f_{0}}\right]$. It is clear by defining

$$
u_{k l}=\sum_{i, j=0}^{k, l}(-1)^{k+l-(i+j)}\left(\begin{array}{l}
k \\
i
\end{array}\right)\left(\begin{array}{l}
l \\
j
\end{array}\right) s^{k-l-i} r^{l-k-j}(r+s)^{i+j} v_{i j}
$$

that $T u=v$ and $u \in \mathscr{B}_{\left[f_{0}\right]}^{r, s}$ for every $k, l \in \mathbb{N}$. So, the map $T$ is surjective. Furthermore, by bearing in mind the following equality

$$
\begin{aligned}
\|u\|_{\mathscr{B}_{\left[f_{0}\right]}^{r, s}} & =\sup _{\rho, \rho^{\prime}, k, l \in \mathbb{N}} \frac{1}{(\rho+1)\left(\rho^{\prime}+1\right)} \sum_{i=k}^{k+\rho} \sum_{j=l}^{l+\rho^{\prime}}\left|\left(B^{(r, s)} u\right)_{i j}\right| \\
& =\sup _{\rho, \rho^{\prime}, k, l \in \mathbb{N}} \frac{1}{(\rho+1)\left(\rho^{\prime}+1\right)} \sum_{i=k}^{k+\rho} \sum_{j=l}^{l+\rho^{\prime}}\left|v_{i j}\right|=\|v\|_{\left[\mathscr{C}_{f_{0}}\right]}
\end{aligned}
$$

that, $T$ preserves the norm. As a result, the assertion of the theorem has been proved.

Theorem 2.4. The inclusion $\mathscr{B}_{\left[f_{0}\right]}^{r, s} \subset \mathscr{B}_{[f]}^{r, s}$ holds.

Proof. Consider any sequence $u=\left(u_{i j}\right) \in \mathscr{B}_{\left[f_{0}\right]}^{r, s}$. In that case, from the relation (2.2), there exists a double sequence $v \in\left[\mathscr{C}_{f_{0}}\right]$ such that $v=\left(v_{k l}\right)=\left(B^{(r, s)} u\right)_{k l}$. Since, $\left[\mathscr{C}_{f_{0}}\right] \subset\left[\mathscr{C}_{f}\right]$, then $v \in\left[\mathscr{C}_{f}\right]$ and this says us that $u \in \mathscr{B}_{[f]}^{r, s}$ which is the desired result.

Theorem 2.5. The inclusion $\mathscr{M}_{u} \subset \mathscr{B}_{\left[f_{0}\right]}^{r, s}$ strictly holds.

Proof. From the inequality

$$
\begin{aligned}
\|u\|_{\mathscr{B}_{\left[f_{0}\right]}^{r, s}} & =\sup _{\rho, \rho^{\prime}, k, l \in \mathbb{N}} \frac{1}{(\rho+1)\left(\rho^{\prime}+1\right)} \sum_{i=k}^{k+\rho} \sum_{j=l}^{l+\rho^{\prime}}\left|\left(B^{(r, s)} u\right)_{i j}\right| \\
& \leq \sup _{\rho, \rho^{\prime}, k, l \in \mathbb{N}} \frac{1}{(\rho+1)\left(\rho^{\prime}+1\right)} \sum_{i=k}^{k+\rho} \sum_{j=l}^{l+\rho^{\prime}}\left|\sum_{m=0}^{i} \sum_{n=0}^{j} b_{i j m n}^{r, s}\right|\left|u_{m n}\right| \\
& \leq \sup _{m, n \in \mathbb{N}}\left|u_{m n}\right| \sup _{\rho, \rho^{\prime}, k, l \in \mathbb{N}} \frac{1}{(\rho+1)\left(\rho^{\prime}+1\right)} \sum_{i=k}^{k+\rho} \sum_{j=l}^{l+\rho^{\prime}}\left|\sum_{m=0}^{i} \sum_{n=0}^{j} b_{i j m n}^{r, s}\right| \\
& =\|u\|_{\infty},
\end{aligned}
$$


it is seen that any double sequence $u$ taken in $\mathscr{M}_{u}$ is in $\mathscr{B}_{\left[f_{0}\right]}^{r, s}$.

Now, let us select the sequence $u=\left(u_{k l}\right)=\frac{(-s-r)^{k+l}}{r^{k} s^{l}}$ to show the strictness. In that case, we see that $u \notin \mathscr{M}_{u}$ but its $B^{(r, s)}$ transform $B^{(r, s)} u=\frac{(-1)^{k+l} r^{k} s^{l}}{(r+s)^{k+l}}$ is in $\mathscr{M}_{u} \cap \mathscr{C}_{P}=\mathscr{C}_{b P} \subset\left[\mathscr{C}_{f_{0}}\right]$ which means that $u \in \mathscr{B}_{\left[f_{0}\right]}^{r, s}$. In the light of all this said, it is seen that $u \in \mathscr{B}_{\left[f_{0}\right]}^{r, s}-\mathscr{M}_{u}$ and the inclusion is strict, as claimed.

Combining Theorem 2.4 and Theorem 2.5, we may give the following corollary:

Corollary 2.6. The inclusion $\mathscr{M}_{u} \subset \mathscr{B}_{[f]}^{r, s}$ strictly holds.

\section{Dual spaces}

In the current section, we deal with the computation of the $\alpha, \beta(b P)$ and $\gamma$-duals of the space $\mathscr{B}_{[f]}^{r, s}$. Before these, let us give some information related duals.

The $\alpha-, \beta(b P)-$ and $\gamma-$ duals of a $\Psi \subset \Omega$ are described as

$$
\begin{aligned}
\Psi^{\alpha} & :=\left\{t=\left(t_{i j}\right) \in \Omega: \sum_{i, j}\left|t_{i j} u_{i j}\right|<\infty \text { for all }\left(u_{i j}\right) \in \Psi\right\}, \\
\Psi^{\beta(b P)} & :=\left\{t=\left(t_{i j}\right) \in \Omega: b P-\sum_{i, j} t_{i j} u_{i j} \text { exists for all }\left(u_{i j}\right) \in \Psi\right\}, \\
\Psi^{\gamma} & :=\left\{t=\left(t_{i j}\right) \in \Omega: \sup _{k, l \in \mathbb{N}}\left|\sum_{i, j=0}^{k, l} t_{i j} u_{i j}\right|<\infty \text { for all }\left(u_{i j}\right) \in \Psi\right\},
\end{aligned}
$$

respectively. It is well known that $\Psi^{\alpha} \subset \Psi^{\gamma}$ and if $\Psi \subset \Lambda$, then $\Lambda^{\alpha} \subset \Psi^{\alpha}$ for the double sequence spaces $\Psi$ and $\Lambda$.

Theorem 3.1. $\left\{\mathscr{B}_{[f]}^{r, s}\right\}^{\alpha}=\mathscr{L}_{u}$.

Proof. To show the inclusion $\left\{\mathscr{B}_{[f]}^{r, s}\right\}^{\alpha} \subset \mathscr{L}_{u}$, assume the sequence $t=\left(t_{k l}\right) \in\left\{\mathscr{B}_{[f]}^{r, s}\right\}^{\alpha}-\mathscr{L}_{u}$. So, $\sum_{k, l}\left|t_{k l} u_{k l}\right|<\infty$ for all $u=\left(u_{k l}\right) \in \mathscr{B}_{[f]}^{r, s}$. If we consider $e=\sum_{k, l} e^{k l}$, we see that $e \in \mathscr{B}_{[f]}^{r, s}$. Since $t e=t \notin \mathscr{L}_{u}$, we obtain from the equality $\sum_{k, l}\left|t_{k l} e\right|=\sum_{k, l}\left|t_{k l}\right|=\infty$ that $t \notin\left\{\mathscr{B}_{[f]}^{r, s}\right\}^{\alpha}$ which is a contradiction. Thus, it must be $t \in \mathscr{L}_{u}$ and the inclusion $\left\{\mathscr{B}_{[f]}^{r, s}\right\}^{\alpha} \subset \mathscr{L}_{u}$ is valid.

For the sufficiency part, let us take the sequences $t=\left(t_{k l}\right) \in \mathscr{L}_{u}$ and $u=\left(u_{k l}\right) \in \mathscr{B}_{[f]}^{r, s}$. Then, there exist a double sequence $v=\left(v_{k l}\right) \in \mathscr{C}_{f}$ with the relation $v_{k l}=\left(B^{(r, s)} u\right)_{k l}$. Since $\mathscr{C}_{f} \subset \mathscr{M}_{u}$, then $\sup _{k, l}\left|v_{k l}\right|<M_{1}$, where $M_{1} \in \mathbb{R}^{+}$. Therefore,

$$
\begin{aligned}
\sum_{k, l}\left|t_{k l} u_{k l}\right| & =\sum_{k, l}\left|t_{k l}\right|\left|\sum_{i, j=0}^{k, l}(-1)^{k+l-(i+j)}\left(\begin{array}{c}
k \\
i
\end{array}\right)\left(\begin{array}{c}
l \\
j
\end{array}\right) s^{k-l-i} r^{l-k-j}(r+s)^{i+j} v_{i j}\right| \\
& \leq \sum_{k, l}\left|t_{k l}\right|\left|\frac{1}{r^{k} s^{l}} \sum_{i, j=0}^{k, l}\left(\begin{array}{c}
k \\
i
\end{array}\right)\left(\begin{array}{c}
l \\
j
\end{array}\right)(-s)^{k-i}(r+s)^{i}(-r)^{l-j}(r+s)^{j}\right|\left|v_{i j}\right| \\
& \leq M_{1} \sum_{k, l}\left|t_{k l}\right|\left|\frac{1}{r^{k} s^{l}} \sum_{i=0}^{k}\left(\begin{array}{c}
k \\
i
\end{array}\right)(-s)^{k-i}(r+s)^{i} \sum_{j=0}^{l}\left(\begin{array}{c}
l \\
j
\end{array}\right)(-r)^{l-j}(r+s)^{j}\right| \\
& =M_{1} \sum_{k, l}\left|t_{k l}\right|
\end{aligned}
$$

and this says us that $t \in\left\{\mathscr{B}_{[f]}^{r, s}\right\}^{\alpha}$. Thus, it is seen that $\mathscr{L}_{u} \subset\left\{\mathscr{B}_{[f]}^{r, s}\right\}^{\alpha}$.

Definition 3.2. [16] A subset $E \subset \mathbb{N}^{+} \times \mathbb{N}^{+}$is said to be uniformly of zero density if and only if the number of elements of $E$ which lie in the rectangle $R$ is $o(\lambda \mu)$ as $\lambda, \mu \rightarrow \infty$, uniformly in $k, l \geq 0$, where $R=\{(i, j): k \leq i \leq k+\lambda-1, \quad l \leq j \leq$ $l+\mu-1\}$ and $\mathbb{N}^{+}=\{1,2,3, \ldots\}$. 
Now, let us describe the sets $w_{1}-w_{7}$ that will be used in calculating $\beta(b P)-$ and $\gamma-$ duals.

$$
\begin{aligned}
& w_{1}=\left\{t=\left(t_{i j}\right) \in \Omega: P-\lim _{k, l \rightarrow \infty} \chi(k, l, i, j, m, n)=0\right\}, \\
& w_{2}=\left\{t=\left(t_{i j}\right) \in \Omega: P-\lim _{k, l \rightarrow \infty} \sum_{i, j} \chi(k, l, i, j, m, n)=1\right\}, \\
& w_{3}=\left\{t=\left(t_{i j}\right) \in \Omega: P-\lim _{k, l \rightarrow \infty} \sum_{i}|\chi(k, l, i, j, m, n)|=0, \quad \forall j \in \mathbb{N}\right\}, \\
& w_{4}=\left\{t=\left(t_{i j}\right) \in \Omega: P-\lim _{k, l \rightarrow \infty} \sum_{j}|\chi(k, l, i, j, m, n)|=0, \quad \forall i \in \mathbb{N}\right\}, \\
& w_{5}=\left\{t=\left(t_{i j}\right) \in \Omega: \exists M_{2}, M_{3} \in \mathbb{N} \ni \sum_{i, j>M_{2}}|\chi(k, l, i, j, m, n)|<M_{3}\right\}, \\
& w_{6}=\left\{t=\left(t_{i j}\right) \in \Omega: b P-\lim _{k, l \rightarrow \infty} \sum_{i \in E} \sum_{j \in E}\left|\triangle_{10} \chi(k, l, i, j, m, n)\right|=0\right\}, \\
& w_{7}=\left\{t=\left(t_{i j}\right) \in \Omega: b P-\lim _{k, l \rightarrow \infty} \sum_{i \in E} \sum_{j \in E}\left|\triangle_{01} \chi(k, l, i, j, m, n)\right|=0\right\},
\end{aligned}
$$

where

$$
\begin{gathered}
\chi(k, l, i, j, m, n)=\sum_{m=i n=j}^{k} \sum^{l}(-1)^{m+n-(i+j)}\left(\begin{array}{c}
m \\
i
\end{array}\right)\left(\begin{array}{c}
n \\
j
\end{array}\right) s^{m-n-i} r^{n-m-j}(r+s)^{i+j} t_{m n}, \\
\triangle_{10} \chi(k, l, i, j, m, n)=\chi(k, l, i, j, m, n)-\chi(k, l, i+1, j, m, n), \\
\triangle_{01} \chi(k, l, i, j, m, n)=\chi(k, l, i, j, m, n)-\chi(k, l, i, j+1, m, n)
\end{gathered}
$$

and $E$ is uniformly of zero density.

Theorem 3.3. $\left\{\mathscr{B}_{[f]}^{r, s}\right\}^{\beta(b P)}=\bigcap_{k=1}^{7} w_{k}$

Proof. Suppose that $t=\left(t_{k l}\right) \in \Omega$ and $u=\left(u_{k l}\right) \in \mathscr{B}_{[f]}^{r, s}$. Thus, $v=\left(v_{k l}\right) \in\left[\mathscr{C}_{f}\right]$ with $B^{(r, s)} u=v$. We obtain by the relation (2.5) that

$$
\begin{aligned}
z_{k l} & =\sum_{i, j=0}^{k, l} t_{i j} u_{i j} \\
& =\sum_{i, j=0}^{k, l} t_{i j}\left\{\sum_{m, n=0}^{i, j}(-1)^{i+j-(m+n)}\left(\begin{array}{c}
i \\
m
\end{array}\right)\left(\begin{array}{c}
j \\
n
\end{array}\right) s^{i-j-m} r^{j-i-n}(r+s)^{m+n} v_{m n}\right\} \\
& =\sum_{i, j=0}^{k, l}\left\{\sum_{m=i n=j}^{k} \sum_{n=1}^{l}(-1)^{m+n-(i+j)}\left(\begin{array}{c}
m \\
i
\end{array}\right)\left(\begin{array}{c}
n \\
j
\end{array}\right) s^{m-n-i} r^{n-m-j}(r+s)^{i+j} t_{m n}\right\} v_{i j} \\
& =\left(O^{r, s} v\right)_{k l}
\end{aligned}
$$

for all $k, l \in \mathbb{N}$, where $O^{r, s}=\left(\begin{array}{c}r, s \\ k l i j\end{array}\right)$ defined by

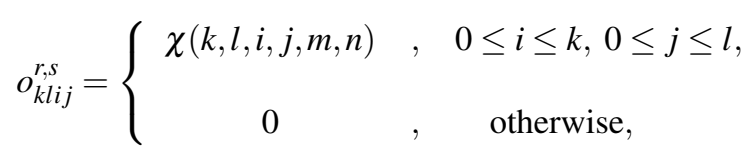

for every $k, l, i, j \in \mathbb{N}$. In that case, by bearing in mind (3.1), it is infered that $t u=\left(t_{k l} u_{k l}\right) \in \mathscr{C} \mathscr{S}_{b P}$ whenever $u=\left(u_{k l}\right) \in \mathscr{B}_{[f]}^{r, s}$ if and only if $z=\left(z_{k l}\right) \in \mathscr{C}_{b P}$ whenever $v=\left(v_{k l}\right) \in\left[\mathscr{C}_{f}\right]$. This implies that $t=\left(t_{k l}\right) \in\left\{\mathscr{B}_{[f]}^{r, s}\right\}^{\beta(b P)}$ if and only if $O^{r, s} \in$ $\left(\left[\mathscr{C}_{f}\right], \mathscr{C}_{b P}\right)$ and the proof is completed in view of Theorem 1 in [16].

Lemma 3.4. [11] A $4 d$ matrix $D=\left(d_{k l i j}\right) \in\left(\left[\mathscr{C}_{f}\right], \mathscr{M}_{u}\right)$ if and only if $D_{k l} \in\left\{\left[\mathscr{C}_{f}\right]\right\}^{\beta(\vartheta)}$ for all $k, l \in \mathbb{N}$ and

$$
\sup _{k, l \in \mathbb{N}} \sum_{i, j}\left|d_{k l i j}\right|<\infty \text {. }
$$


Theorem 3.5. $\left\{\mathscr{B}_{[f]}^{r, s}\right\}^{\gamma}=w_{8} \cap \mathscr{C} \mathscr{S}_{\vartheta}$, where

$$
w_{8}=\left\{t=\left(t_{i j}\right) \in \Omega: \sup _{k, l \in \mathbb{N}} \sum_{i, j}|\chi(k, l, i, j, m, n)|<\infty\right\} .
$$

Proof. We easily reach the proof by the aid of (ii) of Theorem 4.4 in [3]. So, we omit it.

\section{Matrix transformations}

In this part, it will be given the classes $\left(\mathscr{B}_{[f]}^{r, s}, \mathscr{C}_{f}\right)_{\text {reg }}$ and $\left(\mathscr{B}_{[f]}^{r, s}, \mathscr{M}_{u}\right)$. Before these, it is needed to give the following lemma which will be used in Theorem 4.2.

Lemma 4.1. [11] A $4 d$ matrix $D=\left(d_{k l i j}\right) \in\left(\left[\mathscr{C}_{f}\right], \mathscr{C}_{f}\right)_{\text {reg }}$ if and only if $D \in\left(\mathscr{C}_{b P}, \mathscr{C}_{f}\right)_{\text {reg }}$ and $\sum_{i, j \in E}\left|\triangle_{11} d_{k l i j}\right| \rightarrow 0$ as $k, l \rightarrow \infty$ for each set $E$ which is uniformly zero density where

$$
\triangle_{11} d_{k l i j}=d_{k l i j}-d_{k l, i+1, j}-d_{k l i, j+1}+d_{k l, i+1, j+1} .
$$

Theorem 4.2. Consider the $4 d$ infinite matrices $D=\left(d_{k l i j}\right)$ and $H=\left(h_{k l i j}\right)$ whose elements are connected with the equality

$$
h_{k l i j}=\sum_{a=i}^{\infty} \sum_{b=j}^{\infty}\left(\begin{array}{l}
a \\
i
\end{array}\right)\left(\begin{array}{l}
b \\
j
\end{array}\right) s^{a-b-i} r^{b-a-j}(r+s)^{i+j} d_{k l a b} .
$$

In that case, a $4 d$ matrix $D=\left(d_{k l i j}\right) \in\left(\mathscr{B}_{[f]}^{r, s}, \mathscr{C}_{f}\right)_{\text {reg }}$ if and only if

$$
\begin{aligned}
& D_{k l} \in\left\{\mathscr{B}_{[f]}^{r, s}\right\}^{\beta(\vartheta)}, \\
& \sup _{k, l \in \mathbb{N}} \sum_{i, j}\left|h_{k l i j}\right|<\infty, \\
& b P-\lim _{\rho, \rho^{\prime} \rightarrow \infty} \sigma\left(i, j, \rho, \rho^{\prime}, m, n\right)=0, \quad \text { uniformly in } m, n \in \mathbb{N}, \\
& b P-\lim _{\rho, \rho^{\prime} \rightarrow \infty} \sum_{i, j} \sigma\left(i, j, \rho, \rho^{\prime}, m, n\right)=1, \quad \text { uniformly in } m, n \in \mathbb{N}, \\
& b P-\lim _{\rho, \rho^{\prime} \rightarrow \infty} \sum_{i}\left|\sigma\left(i, j, \rho, \rho^{\prime}, m, n\right)\right|=0, \quad \text { uniformly in } m, n \in \mathbb{N}, \\
& b P-\lim _{\rho, \rho^{\prime} \rightarrow \infty} \sum_{j}\left|\sigma\left(i, j, \rho, \rho^{\prime}, m, n\right)\right|=0, \quad \text { uniformly in } m, n \in \mathbb{N}, \\
& \sum_{i, j \in E}\left|\triangle_{11} h_{k l i j}\right| \rightarrow 0, \quad k, l \rightarrow \infty
\end{aligned}
$$

for each set $E$ which is uniformly of zero density where $\sigma\left(i, j, \rho, \rho^{\prime}, m, n\right)=\sum_{k=m}^{m+\rho} \sum_{l=n}^{n+\rho^{\prime}} \frac{h_{k l i j}}{(\rho+1)\left(\rho^{\prime}+1\right)}$.

Proof. Suppose that the matrix $D=\left(d_{k l i j}\right) \in\left(\mathscr{B}_{[f]}^{r, s}, \mathscr{C}_{f}\right)_{r e g}$. Then, $D u$ exists and is in $\mathscr{C}_{f}$ for all $u=\left(u_{k l}\right) \in \mathscr{B}_{[f]}^{r, s}$, which implies that $v=B^{(r, s)} u \in\left[\mathscr{C}_{f}\right]$ and $D_{k l} \in\left\{\mathscr{B}_{[f]}^{r, s}\right\}^{\beta(\vartheta)}$. Thus, condition (4.1) holds. We have the following equality derived from the $(\varsigma, \xi) t h$-partial sums of the series $\sum_{i, j} d_{k l i j} u_{i j}$ by taking into account the relation between the terms of the sequences $u$ and $v$,

$$
\begin{aligned}
\sum_{i, j}^{\varsigma, \xi} d_{k l i j} u_{i j} & =\sum_{i, j}^{\varsigma, \xi} d_{k l i j}\left[\sum_{a, b=0}^{i, j}(-1)^{i+j-(a+b)}\left(\begin{array}{c}
i \\
a
\end{array}\right)\left(\begin{array}{l}
j \\
b
\end{array}\right) s^{i-j-a} r^{j-i-b}(r+s)^{a+b} v_{a b}\right] \\
& =\sum_{i, j}^{\varsigma, \xi}\left[\sum_{a=i}^{\varsigma} \sum_{b=j}^{\xi}(-1)^{a+b-(i+j)}\left(\begin{array}{c}
a \\
i
\end{array}\right)\left(\begin{array}{c}
b \\
j
\end{array}\right) s^{a-b-i} r^{b-a-j}(r+s)^{i+j} d_{k l a b}\right] v_{i j}
\end{aligned}
$$

for all $k, l, m, n \in \mathbb{N}$. Let us define the $4 \mathrm{~d}$ matrix

$$
h_{k l i j}:=\left\{\begin{array}{cc}
\sum_{a=i}^{\infty} \sum_{b=j}^{\infty}(-1)^{a+b-(i+j)}\left(\begin{array}{c}
a \\
i
\end{array}\right)\left(\begin{array}{c}
b \\
j
\end{array}\right) s^{a-b-i} r^{b-a-j}(r+s)^{i+j} d_{k l a b} & 0 \leq i \leq k, 0 \leq j \leq l, \\
0 & , \quad \text { otherwise }
\end{array}\right.
$$


for all $k, l, i, j \in \mathbb{N}$. In that case, by taking $f_{2}$-limit on (4.8) as $\varsigma, \xi \rightarrow \infty$, it is seen that $D u=H v$. Thus, if we take into account the fact that $D \in\left(\mathscr{B}_{[f]}^{r, s}, \mathscr{C}_{f}\right)_{\text {reg }}$ if and only if $H \in\left(\left[\mathscr{C}_{f}\right], \mathscr{C}_{f}\right)_{r e g}$ with Lemma 4.1 and Theorem 3.1 in [39], we can reach the conditions (4.2)-(4.7).

Conversely, from the condition (4.1), Du exists for all $u=\left(u_{k l}\right) \in \mathscr{B}_{[f]}^{r, s}$ such that $v=B^{(r, s)} u \in\left[\mathscr{C}_{f}\right]$ and from (4.8) and (4.9), we see that $D u=H v$. Furthermore, we reach that $H \in\left(\mathscr{C}_{b P}, \mathscr{C}_{f}\right)_{\text {reg }}$ by the aid of the conditions (4.2)-(4.6) and $H \in\left(\left[\mathscr{C}_{f}\right], \mathscr{C}_{f}\right)_{\text {reg }}$ from (4.7). Thus, $D \in\left(\mathscr{B}_{[f]}^{r, s}, \mathscr{C}_{f}\right)_{\text {reg }}$.

Theorem 4.3. A $4 d$ matrix $D=\left(d_{k l i j}\right) \in\left(\mathscr{B}_{[f]}^{r, s}, \mathscr{M}_{u}\right)$ if and only if $D_{k l} \in\left\{\mathscr{B}_{[f]}^{r, s}\right\}^{\beta(\vartheta)}$ for all $k, l \in \mathbb{N}$ and the condition (3.2) holds.

Proof. If we take into account the Lemma 3.4 with the $4 \mathrm{~d}$ matrix $H$ defined in Theorem 4.2 in place of the $4 \mathrm{~d}$ matrix $D$, we can easily reach the proof.

\section{Conclusion}

The concept of matrix domain was examined by several researchers on some single sequence spaces by using some special matrices. As we have mentioned some of them in the current paper, double sequence spaces which are obtained by using the domains of triangular $4 \mathrm{~d}$ matrices have been studied by some authors recently. In the light of these and similar studies, as a natural continuation of the papers [1]-[3], we described two double sequence spaces by using the domain of $4 \mathrm{~d}$ binomial matrix on the spaces of strongly almost convergent and strongly almost null double sequences. Moreover, we investigated their some properties and inclusion relations related them, computed duals and characterized some matrix classes. We conclude that the results obtained from the $4 \mathrm{~d}$ binomial matrix $B^{(r, s)}$ is more general and extensive than the existent results obtained from the 4d Euler matrix $E(r, s)$. We expect that our results might be a reference for further studies in this field.

\section{Acknowledgements}

The authors would like to express their sincere thanks to the editor and the anonymous reviewers for their helpful comments and suggestions.

\section{Funding}

There is no funding for this work.

\section{Availability of data and materials}

Not applicable.

\section{Competing interests}

The authors declare that they have no competing interests.

\section{Author's contributions}

All authors contributed equally to the writing of this paper. All authors read and approved the final manuscript.

\section{References}

[1] S. Demiriz, S. Erdem, On the new double binomial sequence space, Turk. J. Math. Comput. Sci., 12(2) (2020) 101-111.

[2] S. Demiriz, S. Erdem, Domain of binomial matrix in some spaces of double sequences, Punjab Uni. J. Math., 52(11) (2020), 65-79.

[3] S. Erdem, S. Demiriz, Almost convergence and 4-dimensional binomial matrix, Konuralp J. Math., 8(2) (2020), $329-336$.

[4] M. Zeltser, On conservative matrix methods for double sequence spaces, Acta Math. Hung., 95(3) (2002), $225-242$.

[5] M. Zeltser, Investigation of double sequence spaces by soft and hard analytic methods, Ph.D. Thesis, Uni., Tartu, 2001.

[6] B. Altay, F. Başar, Some new spaces of double sequences, J. Math. Anal. Appl., 309(1) (2005), 70-90.

[7] G. Talebi, Operator norms of four-dimensional Hausdorff matrices on the double Euler sequence spaces, Linear and Multilinear Algebra, 65(11) (2017), 2257-2267.

[8] M.Yeşilkayagil, F. Başar, Domain of Euler mean in the space of absolutely p-summable double sequences with $0<p<1$, Anal. Theory Appl., 34(3) (2018), 241-252.

[9] O. Tuğ, Four-dimensional generalized difference matrix and some double sequence spaces, J. Inequal. Appl., 2017(1) (2017), 1-22.

[10] O. Tuğ, On almost B-summable double sequence spaces, J. Inequal. Appl., 2018(1) (2018), 1-19.

[11] O. Tuğ, The spaces of $B(r, s, t, u)$ strongly almost convergent double sequences and matrix transformations, Bull. Sci. Math., 169(2021), 102989.

[12] M.C. Bişgin, The binomial sequence spaces which include the spaces $\ell_{p}$ and $\ell_{\infty}$ and geometric properties, J. Inequal. Appl., 2016 (2016), 304.

[13] M.C. Bişgin, The binomial sequence spaces of nonabsolute type, J. Inequal. Appl., 2016(1) (2016), 1-16.

[14] M.C. Bişgin, The binomial almost convergent and null sequence spaces, Commun. Fac. Sci. Uni. Ank. Series A1, 67(1) (2018), 211-224.

[15] F. Mòricz, B.E. Rhoades, Almost convergence of double sequences and strong regularity of summability matrices, Math. Proc. Camb. Philos. Soc., 104 (1988), 283-294. 
[16] M. Başarır, On the strong almost convergence of double sequences, Period. Math. Hung., 30(3) (1995), $177-181$.

[17] C.R. Adams, On non-factorable transformations of double sequences, Proc. Natl. Acad. Sci. USA, 19(5) (1933), $564-567$.

[18] P.Z. Alp, E.E. Kara, The new class $L_{z, p, E}$ of $s$ - type operators, AIMS Math., 4(3) (2019), 779-791.

[19] F. Başar, Y. Sever, The space $\mathscr{L}_{q}$ of double sequences, Math. J. Okayama Uni., 51 (2009), 149-157.

[20] R.C. Cooke, Infinite Matrices and Sequence Spaces, Macmillan and Co. Limited, London, 1950.

[21] S. Erdem, S. Demiriz, A new RH-regular matrix derived by Jordan's function and its domains on some double sequence spaces, J. Function Spaces, 2021 (2021), Article ID 5594751, 9 pages.

[22] H. J. Hamilton, Transformations of multiple sequences, Duke Math. J., 2 (1936), 29-60.

[23] M. İlkhan, M. A. Bayrakdar, A study on matrix domain of Riesz-Euler totient matrix in the space of p-absolutely summable sequences, Com. Adv. Math. Sci., 4(1) (2021), 14-25.

[24] V.A. Khan, U. Tuba, On paranormed Ideal convergent sequence spaces defined by Jordan totient function, J. Inequal. Appl., 2021(1) (2021), 1-16, https://doi.org/10.1186/s13660-021-02634-7.

[25] M. Kirisci, F. Başar, Some new sequence spaces derived by the domain of generalized difference matrix, Computers and Mathematics with Applications, 60(5) (2010), 1299-1309.

[26] M. Mursaleen, Almost strongly regular matrices and a core theorem for double sequences, J. Math. Anal. Appl., 293(2) (2004), 523-531.

[27] A.K. Noman, E.S. Al Yari, New general results on matrix domains of triangles in sequence spaces, Albaydha Uni. J., 3(2) (2021), 57-72.

[28] F. Nuray, U. Ulusu, E. Dündar, Cesàro summability of double sequences of sets, General Mathematics Notes, 25(1) (2014), 8-18.

[29] F. Nuray, U. Ulusu, Lacunary invariant statistical convergence of double sequences of sets, Creative Math. and Info., 28(2) (2019), 143-150.

[30] F. Nuray, E. Dündar, U. Ulusu, Wijsman statistical convergence of double sequences of set, Iranian J. Math. Sci. Info., 16(1) (2021), 55-64.

[31] A. Pringsheim, Zur Theorie der zweifach unendlichen Zahlenfolgen, Math. Ann., 53 (1900), 289-321.

[32] G. M. Robison, Divergent double sequences and series, Amer. Math. Soc. Trans., 28 (1926), 50-73.

[33] O. Tuğ, V. Rakočević, E. Malkowsky, On the domain of the four-dimensional sequential band matrix in some double sequence Spaces, Mathematics, 8(5)(2020), 789.

[34] O. Tuğ, The spaces of B $(r, s, t, u)$ strongly almost convergent double sequences and matrix transformations, Bull. Sci. Math., 169 (2021), 102989.

[35] T. Yaying, B. Hazarika, On sequence spaces generated by binomial difference operator of fractional order, Math. Slovaca, 69(4) (2019), 901-918.

[36] M.Yeşilkayagil, F. Başar, Some topological properties of the spaces of almost null and almost convergent double sequences, Turk. J. Math., 40(3) (2016), 624-630.

[37] M.Yeşilkayagil, F. Başar, On the characterization of a class of four dimensional matrices and Steinhaus type theorems, Kragujevac J. Math., 40(1)(2016), $35-45$

[38] M.Yeşilkayagil, F. Başar, Domain of Riesz mean in the space $\mathscr{L}_{p}$, Filomat, 31(4) (2017), 925-940.

[39] M. Zeltser, M. Mursaleen, S. A. Mohiuddine, On almost conservative matrix methods for double sequence spaces, Publ. Math. Debrecen, 75 (2009), 387-399. 